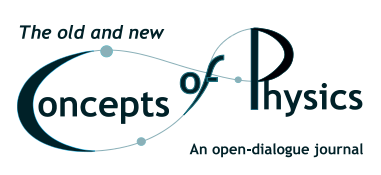

www.uni.lodz.pl/concepts

\title{
A SIMPLE MODEL FOR AN ULTRACOLD PLASMA
}

\author{
S. Sevinçli, M. Ö. Oktel, and B. Tanatar \\ Bilkent University, Department of Physics \\ 06800 Bilkent, Ankara, Turkey \\ e-mail: oktel@fen.bilkent.edu.tr
}

(Received 18 December 2007; accepted 1 March 2007)

\begin{abstract}
We consider an ultracold plasma composed of the same number of electrons and bosonic ions, at zero temperature. We calculate the ion and electron densities taking the long range interactions into account using a modified Thomas-Fermi approximation. We assume that ions are trapped by harmonic trap while electrons are only trapped by the ion cloud. For a cloud that contains $10^{4}$ electrons and $10^{4}$ ions confined with a trap frequency of $10 \mathrm{KHz}$, we find that stable radius is around $14 \mu m$.
\end{abstract}




\section{S. Sevinçli, M. Ö. Oktel, and B. Tanatar}

\section{Introduction}

As a consequence of rapid development in cooling and trapping mechanisms, number of experiments on ultracold systems increase and open new directions every day. One of these new directions is the creation of an ultracold plasma. Ultracold plasma is created by photoionization of atoms in a magneto-optical trap using a pulsed laser [1]. The initial observation was made with Xe atoms, but since then similar results have been found with $\mathrm{Rb}, \mathrm{Cs}$, and $\mathrm{Sr}$ atoms $[2],[3],[4]$.

A lot of interesting phenomena has been observed in ultracold plasmas, such as expansion of the plasma and recombination to form Rydberg atoms, both of which occur on the time scale of tens of microseconds. After photoionization, electrons carry the most of the energy since they are very light, and ions stay almost as cold as the initial atoms.A small fraction of the electrons leave the gas, the excess positive charge of the ions traps the remaining electrons, forming a quasi-neutral plasma [4]-[11].The ions and electrons thermalize among themselves, however as the plasma is not trapped, they do not thermalize with each other within the time scale of the experiment. Dynamical properties of the system can be investigated by understanding the thermalization of the plasma.

After the creation of an ultracold plasma the evolution of cold Rydberg atoms into a plasma was observed [3]-[16]. Cold dense samples of Rydberg atoms are fascinating since they combine the atomic physics, plasma physics, and solid state physics. Ultracold plasma formation from a Bose condensed gas was also observed [2].

The electron Coulomb coupling parameter, $\Gamma \approx e^{2} n^{1 / 3} / k_{B} T$, is a measure of how strongly coupled the plasma is. For an ultracold plasma, this parameter is generally about one. A plasma becomes strongly coupled when the electrical interaction energy between charged particles exceeds the thermal energy, i.e. $\Gamma \gg 1$. In this regime, recombination, collective modes, and thermalization are interesting properties to investigate. However, it is still a challenge to reach this regime experimentally. One idea would be to design an optical trap that would couple to the ions, which subsequently would trap the electrons and form a stable system.

In a previous work[17], we studied in simple model of quantum degenerate plasma where a quadratic trap for the ions have been set 
up. We investigated the dependence of the cloud size on electron density by using a Gaussian variational wave function. We assumed that there is a constant density of electrons in the cloud giving rise to screened ion-ion interactions [18].

In this paper, we again study the same system, however the density of electrons is not assumed to be a constant but used as a dynamical variable. We calculate the densities of ions and electrons using a modified Thomas-Fermi model [19]. We find that the expression for the size of the cloud obtained in our previous work is in good agreement with the current more complicated model.

Our method enables us to calculate the ion and electron densities separately, and as a result we find that the equilibrium structure is a plasma which is neutral throughout. Thus the perfect screening property of plasma is valid for even an ultracold degenerate system.

\section{Theoretical Model}

The ion cloud is described as a Bose condensed system within the mean-field approximation. The ground state energy functional at zero temperature is given by

$$
\begin{aligned}
E_{i}[\psi(\mathbf{r})] & =\int d \mathbf{r}\left\{\frac{\hbar^{2}}{2 m_{i}}|\nabla \psi(\mathbf{r})|^{2}+\frac{1}{2} m_{i} \omega^{2} r^{2}|\psi(\mathbf{r})|^{2}\right\}+ \\
& +Z^{2} e^{2} \iint d \mathbf{r} d \mathbf{r}^{\prime} \frac{|\psi(\mathbf{r})|^{2}\left|\psi\left(\mathbf{r}^{\prime}\right)\right|^{2}}{\left|\mathbf{r}-\mathbf{r}^{\prime}\right|}- \\
& -Z e^{2} \iint d \mathbf{r} d \mathbf{R} \frac{|\psi(\mathbf{r})|^{2} n(\mathbf{R})}{|\mathbf{r}-\mathbf{R}|}
\end{aligned}
$$

where $\psi(\mathbf{r})$ is the condensate wave function, $\omega$ is the harmonic trap frequency, and $n(\mathbf{r})$ is the electron density. We assume that bosonic ions and electrons interact via the bare Coulomb potential. Energy functional is minimized by the condition

$$
\delta\left\{E_{i}-\mu_{i} \int|\psi(\mathbf{r})|^{2} d \mathbf{r}\right\}=0
$$




\section{S. Sevinçli, M. Ö. Oktel, and B. Tanatar}

where $\mu_{i}$ the chemical potential related to the normalization condition $N_{i}=\int|\psi(\mathbf{r})|^{2} d \mathbf{r}$. The Gross-Pitaevskii equation becomes

$$
\begin{array}{r}
{\left[-\frac{\hbar^{2}}{2 m_{i}} \nabla^{2}+\frac{1}{2} m_{i} \omega r^{2}+Z^{2} e^{2} \int d \mathbf{r}^{\prime} \frac{\left|\psi\left(\mathbf{r}^{\prime}\right)\right|^{2}}{\left|\mathbf{r}-\mathbf{r}^{\prime}\right|}\right.} \\
\left.-Z e^{2} \int d \mathbf{R} \frac{n(\mathbf{R})}{|\mathbf{r}-\mathbf{R}|}\right] \psi(\mathbf{r})=\mu_{i} \psi(\mathbf{r}) .
\end{array}
$$

The Thomas-Fermi energy functional for electrons is composed of three terms,

$$
\begin{aligned}
E^{T F}[n(\mathbf{r})]=T^{T F}[n(\mathbf{r})]-Z e^{2} & \iint d \mathbf{r} d \mathbf{R} \frac{n(\mathbf{r})|\psi(\mathbf{R})|^{2}}{|\mathbf{r}-\mathbf{R}|}+ \\
& +\frac{e^{2}}{2} \iint d \mathbf{r} d \mathbf{r}^{\prime} \frac{n(\mathbf{r}) n\left(\mathbf{r}^{\prime}\right)}{\left|\mathbf{r}-\mathbf{r}^{\prime}\right|} .
\end{aligned}
$$

The first term is the kinetic energy and obtained by integrating the kinetic energy density a homogeneous electron gas,

$$
T^{T F}[n(\mathbf{r})]=\int d \mathbf{r} t_{0}[n(\mathbf{r})],
$$

where $t_{0}[n(\mathbf{r})]$ is obtained by summing all of the free energy states $\varepsilon=\hbar^{2} k^{2} / 2 m_{e}$ up to the Fermi wave vector $k_{F}=\left[3 \pi^{2} n(\mathbf{r})\right]^{1 / 3}$.

$$
t_{0}[n(\mathbf{r})]=\frac{\hbar^{2}}{2 \pi^{2} m_{e}} \int_{0}^{k_{F}} k^{4} d k=\frac{3 \hbar^{2}}{10 m_{e}}\left(3 \pi^{2}\right)^{2 / 3} n(\mathbf{r})^{5 / 3}
$$

then the kinetic energy is obtained as

$$
T^{T F}[n(\mathbf{r})]=\frac{3 \hbar^{2}}{10 m_{e}}\left(3 \pi^{2}\right)^{2 / 3} \int d \mathbf{r} n(\mathbf{r})^{5 / 3} .
$$

The second term is the electrostatic energy of attraction between the ions and the electrons and the last term represents the electronelectron interactions in the system which is approximated by the Coulomb repulsion between electrons. There is no interaction between the electrons and the harmonic trap. Then the Thomas-Fermi energy functional becomes

$$
\begin{aligned}
& E^{T F}[n(\mathbf{r})]=\frac{3 \hbar^{2}}{10 m_{e}}\left(3 \pi^{2}\right)^{2 / 3} \int d \mathbf{r} n(\mathbf{r})^{5 / 3}- \\
& -Z e^{2} \iint d \mathbf{r} d \mathbf{R} \frac{n(\mathbf{r})|\psi(\mathbf{R})|^{2}}{|\mathbf{r}-\mathbf{R}|}+\frac{e^{2}}{2} \iint d \mathbf{r} d \mathbf{r}^{\prime} \frac{n(\mathbf{r}) n\left(\mathbf{r}^{\prime}\right)}{\left|\mathbf{r}-\mathbf{r}^{\prime}\right|}
\end{aligned}
$$




\section{A simple model for an ultracold plasma}

The Thomas-Fermi equation is obtained by minimizing the energy functional by using

$$
\delta\left\{E^{T F}-\mu_{e} \int n(\mathbf{r}) d \mathbf{r}\right\}=0
$$

and $N_{e}=\int n(\mathbf{r}) d \mathbf{r}$,

$$
\frac{\hbar^{2}}{2 m_{e}}\left(3 \pi^{2}\right)^{2 / 3} n(\mathbf{r})^{2 / 3}-Z e^{2} \int d \mathbf{R} \frac{|\psi(\mathbf{R})|^{2}}{|\mathbf{r}-\mathbf{R}|}+e^{2} \int d \mathbf{r}^{\prime} \frac{n\left(\mathbf{r}^{\prime}\right)}{\left|\mathbf{r}-\mathbf{r}^{\prime}\right|}=\mu_{e} .
$$

The total energy functional becomes

$$
\begin{aligned}
E[\psi(\mathbf{r}), n(\mathbf{r})] & =\int d \mathbf{r}\left\{\frac{\hbar^{2}}{2 m_{i}}|\nabla \psi(\mathbf{r})|^{2}+\frac{1}{2} m_{i} \omega^{2} r^{2}|\psi(\mathbf{r})|^{2}\right\}+ \\
& +Z^{2} e^{2} \iint d \mathbf{r} d \mathbf{r}^{\prime} \frac{|\psi(\mathbf{r})|^{2}\left|\psi\left(\mathbf{r}^{\prime}\right)\right|^{2}}{\left|\mathbf{r}-\mathbf{r}^{\prime}\right|}- \\
& -Z e^{2} \iint d \mathbf{r} d \mathbf{R} \frac{|\psi(\mathbf{r})|^{2} n(\mathbf{R})}{|\mathbf{r}-\mathbf{R}|}+ \\
& +\frac{3 \hbar^{2}}{10 m_{e}}\left(3 \pi^{2}\right)^{2 / 3} \int d \mathbf{r} n(\mathbf{r})^{5 / 3}+ \\
& +\frac{e^{2}}{2} \iint d \mathbf{r} d \mathbf{r}^{\prime} \frac{n(\mathbf{r}) n\left(\mathbf{r}^{\prime}\right)}{\left|\mathbf{r}-\mathbf{r}^{\prime}\right|}
\end{aligned}
$$

Dimensionless units are introduced by making the following transformation: (i) $\mathbf{r} \rightarrow l \mathbf{r}$, where $l=\sqrt{\hbar / m_{i} \omega}$ is oscillator length, and (ii) the energy is measured in units of $\hbar \omega$.

The energy functional can now be rewritten using the dimensionless quantities

$$
\begin{aligned}
& \frac{E}{\hbar \omega}=\frac{1}{2} \int d \mathbf{r}\left\{|\nabla \psi(\mathbf{r})|^{2}+r^{2}|\psi(\mathbf{r})|^{2}+\frac{3\left(3 \pi^{2}\right)^{2 / 3}}{5} \frac{m_{i}}{m_{e}} n(\mathbf{r})^{5 / 3}\right\}+ \\
& +\gamma \iint d \mathbf{r} d \mathbf{r}^{\prime}\left\{\frac{|\psi(\mathbf{r})|^{2}\left|\psi\left(\mathbf{r}^{\prime}\right)\right|^{2}}{\left|\mathbf{r}-\mathbf{r}^{\prime}\right|}-\frac{1}{Z} \frac{|\psi(\mathbf{r})|^{2} n\left(\mathbf{r}^{\prime}\right)}{\left|\mathbf{r}-\mathbf{r}^{\prime}\right|}+\frac{1}{2 Z^{2}} \frac{n(\mathbf{r}) n\left(\mathbf{r}^{\prime}\right)}{\left|\mathbf{r}-\mathbf{r}^{\prime}\right|}\right\}
\end{aligned}
$$

where $\gamma=\frac{(Z e)^{2} \sqrt{\frac{m_{i}}{\hbar \omega}}}{\hbar}$ is the electrostatic coupling constant. Similarly,

$$
\left[-\nabla^{2}+r^{2}+2 \gamma \int d \mathbf{r}^{\prime} \frac{\left|\psi\left(\mathbf{r}^{\prime}\right)\right|^{2}}{\left|\mathbf{r}-\mathbf{r}^{\prime}\right|}-\frac{2 \gamma}{Z} \int d \mathbf{r}^{\prime} \frac{n\left(\mathbf{r}^{\prime}\right)}{\left|\mathbf{r}-\mathbf{r}^{\prime}\right|}\right] \psi(\mathbf{r})=2 \mu_{i} \psi(\mathbf{r})
$$


and

$$
\frac{\left(3 \pi^{2}\right)^{2 / 3}}{2} \frac{m_{i}}{m_{e}} n(\mathbf{r})^{2 / 3}-\frac{\gamma}{Z} \int d \mathbf{r}^{\prime} \frac{\left|\psi\left(\mathbf{r}^{\prime}\right)\right|^{2}}{\left|\mathbf{r}-\mathbf{r}^{\prime}\right|}+\frac{\gamma}{Z^{2}} \int d \mathbf{r}^{\prime} \frac{n\left(\mathbf{r}^{\prime}\right)}{\left|\mathbf{r}-\mathbf{r}^{\prime}\right|}=\mu_{e}
$$

are the equations satisfied by condensate wavefunction $\psi\left(\mathbf{r}^{\prime}\right)$ and electron density $n\left(\mathbf{r}^{\prime}\right)$.

To find the density distributions, we employed a modified ThomasFermi model [19]. We assumed spherically symmetric density distributions. If the electrostatic potential arises from electron-ion and ion-ion interactions is called $\phi$, the equations of motion become

$$
\frac{\left(3 \pi^{2}\right)^{2 / 3}}{2} \frac{m_{i}}{m_{e}} n(\mathbf{r})^{2 / 3}-\frac{\gamma}{Z} \phi=\mu_{e}
$$

and

$$
\frac{r^{2}}{2}+\gamma \phi=\mu_{i}
$$

Substituting $\phi$ into Eq. (15) the electron density is obtained as

$$
n(\mathbf{r})=\frac{1}{3 \pi^{2}}\left[\frac{2 m_{e}}{Z m_{i}}\right]^{3 / 2}\left[Z \mu_{e}+\mu_{i}-\frac{r^{2}}{2}\right]^{3 / 2} .
$$

Making use of the Poisson equation

$$
\nabla^{2} \phi=-4 \pi\left[|\psi(\mathbf{r})|^{2}-n(\mathbf{r}) / Z\right]
$$

one gets the ion density as

$$
|\psi(\mathbf{r})|^{2}=\frac{1}{3 \pi^{2} Z}\left[\frac{2 m_{e}}{Z m_{i}}\right]^{3 / 2}\left[Z \mu_{e}+\mu_{i}-\frac{r^{2}}{2}\right]^{3 / 2}+\frac{3}{4 \pi \gamma} .
$$

Charge neutrality of the plasma requires $Z e N_{i}-e N_{e}=0$. After normalization we get

$$
n(\mathbf{r})=\frac{2 \sqrt{2} N_{e}}{\pi^{2}\left(Z \mu_{e}+\mu_{i}\right)^{3}}\left[Z \mu_{e}+\mu_{i}-\frac{r^{2}}{2}\right]^{3 / 2}
$$

and

$$
|\psi(\mathbf{r})|^{2}=\frac{N_{i}\left\{\frac{1}{3 \pi^{2} Z}\left[\frac{2 m_{e}}{Z m_{i}}\right]^{3 / 2}\left[Z \mu_{e}+\mu_{i}-\frac{r^{2}}{2}\right]^{3 / 2}+\frac{3}{4 \pi \gamma}\right\}}{\left[\frac{\left(Z \mu_{e}+\mu_{i}\right)^{3}}{6 \sqrt{2} Z}\left[\frac{2 m_{e}}{Z m_{i}}\right]^{3 / 2}+\frac{2^{3 / 2}\left(Z \mu_{e}+\mu_{i}\right)^{3 / 2}}{\gamma}\right]} .
$$


A simple model for an ultracold plasma

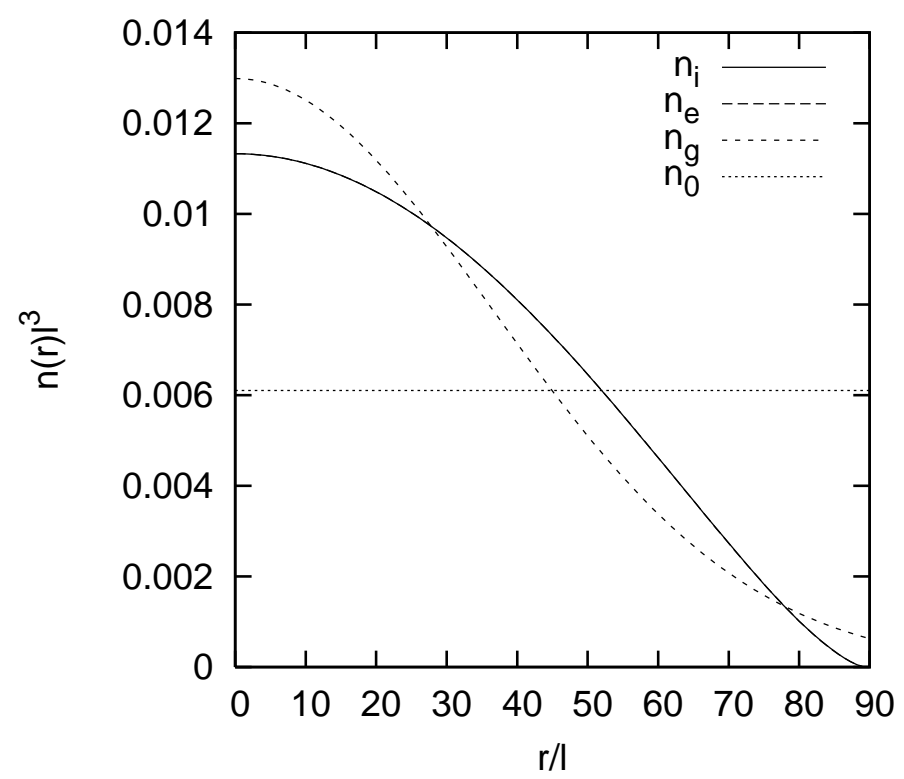

Figure 1: Density distributions as a function of $r / l$ where $l$ is oscillator length. Coupling parameter $\gamma=10^{8}$ and $N_{e}=N_{i}=10^{4}$. Gaussian density for the ions $n_{g}=$ and constant electron density $n_{0}$ used in the previous work [17] is also shown.

Figure 1 shows the density distributions for electrons and ions for $N_{e}=N_{i}=10^{4}$ and coupling parameter $\gamma=10^{8}$. Gaussian density for the ions and constant density for electron that we used in the previous work are shown in the figure also. One can see that electron and ion densities are completely the same. This implies the complete screening of the plasma.

We find the cloud size for the system by using the same parameters that we used before. The cloud size becomes approximately $14 \mu \mathrm{m}$ for $10^{4}$ electrons and $10^{4}$ ions confined with a trapping frequency 10 $\mathrm{KHz}$. 


\section{Conclusion}

We have calculated the size of the cloud, assuming that ions are Bose condensed and confined in a quadratic trap. This method is more accurate than that we used in a previous work, since we did not use the constant electron density and gaussian trial wave function for the ions. Instead of using variational method we calculated the electron and ion densities by means of Thomas-Fermi model. Although the variational model is not so accurate, the result obtained from this model is in good agreement with the current result.

We see that complete screening of plasma is obvious in this system since ion and electron densities are completely the same. For $10^{4}$ ions and $10^{4}$ electrons cloud size is found as $14 \mu \mathrm{m}$ when the order of the trapping frequency is $10 \mathrm{KHz}$.

\section{References}

[1] T. C. Killian, S. Kulin, s. D. Bergeson, L. A. Orozco, C. Orzel, S. L. Rolston, Phys. Rev. Lett. 83, 4776 (1999).

[2] D. Ciampini, M. Anderlini, J. H. Mller, F. Fuso, O. Morsch, J. W. Thomsen, E. Arimondo, Phys. Rev. A 66, 043409 (2002).

[3] T. F. Gallagher, P. Pillet, M. P. Robinson, B. Laburthe-Tolra, M. W. Noel, J. Opt. Soc. Am. B 20, 1091 (2003).

[4] C. E. Simien, Y. C. Chen, P. Gupta, S. Laha, Y. N. Martinez, P. G. Mickelson, S. B. Nagel, T. C. Killian, Phys. Rev. Lett. 92, 143001 (2004).

[5] T. C. Killian, Nature 429, 815 (2004).

[6] T. Pohl, T. Pattard, J. M. Rost, Phys. Rev. A 70, 033416 (2004).

[7] T. C. Killian, V. S. Ashoka, P. Gupta, S. Laha, S. B. Nagel, C. E. Simien, S. Kulin, S. L. Rolston, S. D. Bergeson, J. Phys. A 36, 6077 (2003).

[8] S. D. Bergeson and R. L. Spencer, Phys. Rev. E 67, 026414 (2003).

[9] A. N. Tkachev and S. I. Yakovlenko , JETP Lett. 73, 66 (2001). 


\section{A simple model for an ultracold plasma}

[10] S. Kulin, T. C. Killian, S. D. Bergeson, S. L. Rolston, Phys. Rev. Lett. 85, 318 (2000).

[11] L. I. Minshikov and P. O. Fedichev , Z. Eksp. T. Fiziki.,108, 144 (1995).

[12] A. N. Tkachev and S. I. Yakovlenko, Quantum Electron. 31, 1084 (2001).

[13] T. C. Killian, M. J. Lim, S. Kulin, R. Dumke, S. D. Bergeson, S. L. Rolston, Phys. Rev. Lett. 86, 3759 (2001).

[14] T. Pohl, T. Pattard, J. M. Rost, Phys. Rev. A 68, 010703 (2003).

[15] S. K. Dutta, D. Feldbaum, A. Walz-Flannigan, J. R. Guest, G. Raithel, Phys. Rev. Lett. 86, 3993 (2001).

[16] M. P. Robinson, B. L. Tolra, M. W. Noel, T. F. Gallagher, P. Pillet, Phys. Rev. Lett. 85, 4466 (2000).

[17] S. Sevinçli, M. Ö. Oktel, and B. Tanatar, Phys. Lett. A 350, 129 (2006)

[18] C. Kittel, Introduction to Solid State Physics, (Wiley, New York, 1996).

[19] L. D. Landau, E. M. Lifshitz, Quantum Mechanics, (Pergamon, Oxford, 1994). 